\title{
Comparação entre usuários de crack e de outras drogas em serviço ambulatorial especializado de hospital universitário
}

\author{
Renata Werneck Vargens ${ }^{1}$ \\ Marcelo Santos Cruz ${ }^{2}$ \\ Manoel Antônio dos Santos ${ }^{3}$
}

\begin{abstract}
O crescimento do consumo de crack e o perfil dos usuários no Brasil estão bem documentados, mas faltam estudos na cidade do Rio de Janeiro. Conhecer o perfil sociodemográfico e padrão do uso de drogas desses usuários é fundamental para direcionar recursos, efetivamente. Foram estudados pacientes acolhidos no Programa de Estudos e Assistência ao Uso Indevido de Drogas (Projad) do Instituto de Psiquiatria da Universidade Federal do Rio de Janeiro, entre março de 2007 e setembro de 2008, e analisadas as diferenças entre pacientes com e sem relato de uso de crack na vida. Usuários de crack são significativamente mais jovens e solteiros. Apesar do predomínio de baixa escolaridade e desemprego, esses resultados não foram significativos. Houve crescimento de usuários de crack que procuraram tratamento. Este é o primeiro estudo científico apontando a expansão do consumo de crack no Rio de Janeiro e pode subsidiar a implementação de políticas públicas.
\end{abstract}

Descritores: Cocaína Crack; Transtornos Relacionados ao Uso de Substâncias; Pacientes; Estudos Transversais.

\footnotetext{
${ }_{1}^{1}$ Médica Psiquiatra, Instituto de Psiquiatria, Universidade Federal do Rio de Janeiro, RJ, Brasil. E-mail: revargens@uol.com.br.

2 Médico, Doutor em Psiquiatria, Instituto de Psiquiatria, Universidade Federal do Rio de Janeiro, RJ, Brasil. E-mail: marceloscruz@terra.com.br.

${ }^{3}$ Psicólogo, Doutor em Psicologia. Professor Doutor, Faculdade de Filosofia, Ciências e Letras de Ribeirão Preto, Universidade de São Paulo, SP, Brasil. E-mail: masantos@ffclrp.usp.br.
}

Correspondencia:

Manoel Antônio dos Santos

Universidade de São Paulo. Faculdade de Filosofia, Ciências e Letras de Ribeirão Preto.

Av. dos Bandeirantes, 3900

Bairro: Monte Alegre

CEP: 14040-902 Ribeirão Preto, SP, Brasil

E-mail: masantos@ffclrp.usp.br 


\title{
Comparación entre usuarios de crack y de otras drogas en un servicio ambulatorio de un hospital universitario
}

\begin{abstract}
A pesar de existir investigaciones sobre el crecimiento del consumo de crack y el perfil de sus usuarios en Brasil, no hay estudios específicos en la ciudad de Rio de Janeiro. Ese estudio es importante para dirigir los recursos efectivamente. Los pacientes del Programa de Estudios y Asistencia de la Drogadicción (PROJAD) del Instituto de Psiquiatría de la Universidad Federal de Rio de Janeiro entre marzo de 2007 y septiembre de 2008 fueron estudiados y las diferencias entre pacientes con y sin uso de crack fueran analizadas. Los usuarios de crack son significativamente más jóvenes y solteros. A pesar del predominio del bajo nivel de educación y desempleo, esos resultados no son significativos. Se constata un crecimiento de usuarios de crack que procuran tratamiento. Este es el primer estudio científico que presenta el crecimiento del consumo de crack en Rio de Janeiro y puede servir como subsidio en la implementación de políticas públicas.

Descriptores: Cocaína Crack; Trastornos Relacionados con Sustancias; Pacientes; Estudios Transversales.
\end{abstract}

\section{Comparison Between Crack and Other Drugs Abusers in a Specialized Outpatient Facility of a University Hospital}

The increase in crack abuse and the profile of these abusers are well documented in Brazil, but studies in Rio de Janeiro are lacking. Optimization of resource allocation for prevention and treatment requires a better characterization of abusers with respect to socio-demographic characteristics and drugs use pattern. Patients admitted to the Drug Abuse Research and Care Program (PROJAD) at the Psychiatric Institute of the Federal University of Rio de Janeiro between March 2007 and September 2008 were studied. Crack abusers were found to be significantly younger than other drug abusers and more frequently unmarried. Lower education level and unemployment were a trend in this group, although not statistically significant. The increase in the number of patients seeking treatment for crack abuse was documented. This is the first scientific study reporting on the expansion of crack abuse in Rio de Janeiro, which may provide information to put in practice public policies.

Descriptors: Crack Cocaine; Substance-Related Disorders; Patients; Cross-Sectional Studies.

\section{Introdução}

A despeito da quantidade expressiva de estudos no campo do uso de álcool e outras drogas, o consumo de substâncias psicoativas é problema mundial(1-2). O consumo de drogas no Brasil não destoa do que vem ocorrendo, via de regra, em escala mundial. De acordo com os levantamentos domiciliares nacionais, realizados pelo Centro Brasileiro de Informações sobre Drogas Psicotrópicas (Cebrid), em 2001 e 2005(3-4), o consumo no país de qualquer droga ilícita, na vida aumentou, de 19,4 para $22,8 \%$ e a estimativa de dependentes de álcool, de 11,2 para 12,3\%.
O uso de crack difundiu-se nos Estados Unidos, a partir de meados da década de 1980, e na Europa, no início dos anos 1990, e tem sido preocupação crescente para a comunidade internacional ${ }^{(1,5-6)}$. No Brasil, o uso de crack se iniciou no final dos anos 1980 e seu consumo aumentou rapidamente, nos anos seguintes, devido ao preço baixo e aos efeitos mais intensos dessa forma de consumo ${ }^{(7-8)}$.

O uso do crack ao longo da vida no país, estimado pelos dois últimos levantamentos nacionais, aumentou de 0,4\%, em 2001, para 0,7\%, em 2005(3-4). 
Especificamente na Região Sudeste, o uso de crack na vida aumentou de 0,4 para $0,9 \%{ }^{(3-4)}$. Já existem estudos brasileiros a respeito dessa questão(7-10). A introdução do crack no Rio de Janeiro, indicada em jornais e noticiários(11-12), é relativamente recente, se comparada com outras metrópoles brasileiras. No entanto, não se encontra, em periódicos científicos, o relato do aumento do consumo de crack nessa cidade.

O Programa de Estudos e Assistência ao Uso Indevido de Drogas (Projad) é um serviço ambulatorial de hospital universitário terciário que tem como proposta atender pacientes com transtornos relacionados ao uso de substâncias psicoativas e está aberto à população, sem necessidade de encaminhamento específico. Para atender essa demanda, a partir do ano 2007, a admissão no serviço passou a se dar por meio de grupo de acolhimento, composto por até oito pacientes acima de 18 anos, coordenado por médico psiquiatra e um psicólogo. Esse grupo vem funcionando, desde então, com interrupção entre novembro de 2007 e janeiro de 2008, por razões administrativas.

Após a realização do grupo de acolhimento, o paciente pode ser encaminhado para outras instituições, ou para dar continuidade ao tratamento em um ou mais dos dispositivos do Projad. Essa decisão é tomada observando-se o quadro clínico e a demanda do paciente, quer os usuários venham espontaneamente ou sejam encaminhados pela Justiça para visita ao local. Quando há necessidade de atendimento de emergência ou internação, o paciente é encaminhado para instituições da rede. O serviço segue as orientações de regionalização do SUS, sem perder de vista que se trata de hospital terciário, com equipe técnica ciente das dificuldades sociais, econômicas e administrativas do município e suas adjacências. O serviço atende pacientes com comorbidades psiquiátricas, desde que o problema relacionado ao uso de substâncias psicoativas seja preponderante, no caso.

Mesmo com mudanças recentes na política pública, concernente a problemas relacionados a álcool e outras drogas, ainda se observa escassez de serviços especializados nesse tipo de atendimento. Conhecer as características dos usuários desses serviços possibilita fundamentar propostas para melhor adequação dos dispositivos de tratamento e otimizar os recursos disponíveis.

Neste estudo, consideraram-se pacientes acolhidos todos aqueles que procuraram o serviço e participaram do grupo de acolhimento, e pacientes elegíveis, o subgrupo constituído por aqueles que, depois de participarem do grupo, foram encaminhados para atendimento médico e/ou psicoterápico nesse serviço. Será utilizado o termo "retorno" para definir o comparecimento no serviço em pelo menos um dos atendimentos propostos, no grupo de acolhimento.

O objetivo principal deste estudo foi identificar possíveis diferenças sociodemográficas entre pacientes com e sem história de uso de crack na vida, em um serviço ambulatorial de hospital universitário especializado em problemas relacionados a substâncias psicoativas. Este estudo também se propôs a avaliar a distribuição dos usuários de crack ao longo do período do estudo.

\section{Método}

Trata-se de estudo transversal descritivo, cuja população incluiu todos os pacientes que buscaram atendimento no Projad desde o início da nova forma de acolhimento, instituída em março de 2007 até setembro de 2008, quando se encerrou o período de coleta de dados.

Foram utilizados como fonte de dados as fichas de acolhimento, o livro de registro do grupo de acolhimento e dados de prontuários. As variáveis estudadas foram: sexo, idade, ocupação, escolaridade, local de moradia, tratamentos prévios e participação anterior em grupos de mútua ajuda (Alcoólicos Anônimos - AA, ou Narcóticos Anônimos - NA) e drogas consumidas na vida e nos últimos 30 dias. A região de moradia foi analisada, segundo a divisão da cidade em suas áreas programáticas, conforme divisão do Sistema Único de Saúde, a partir do endereço constante na ficha de acolhimento. O Projad encontra-se na área programática 2.1. Pacientes cuja ocupação referida era "estudante", para efeitos de análise, foram considerados no campo ocupacional como "ativos".

Os dados coletados foram colocados em banco de dados e revisados. A análise dos dados se deu por meio do programa estatístico SPSS, versão 13.0, mediante realização de testes de frequência simples, percentual e teste qui-quadrado. O nível de significância adotado foi $p \leq 0,05$.

\section{Resultados}

Dos 293 pacientes acolhidos, foram localizadas as fichas de acolhimento de 278, correspondendo à perda de $5 \%$ dos indivíduos acolhidos. Entre os pacientes analisados, em alguns casos não foi possível identificar a elegibilidade para o tratamento ou o uso de crack na vida, conforme mostra a Figura 1. 


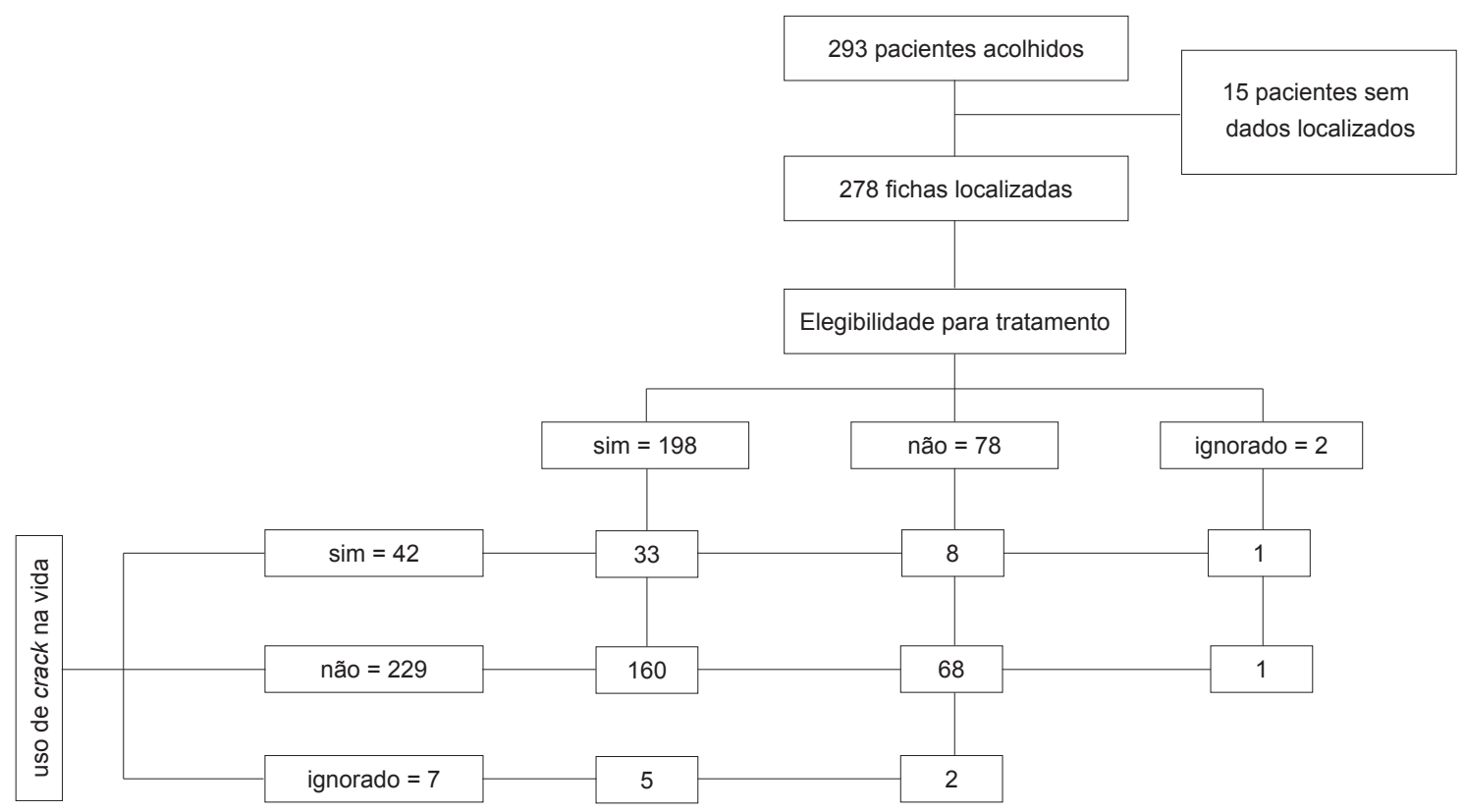

Figura 1 - Fluxograma da distribuição dos pacientes acolhidos, de acordo com elegibilidade para tratamento e uso de crack na vida. Cidade do Rio de Janeiro, RJ, Brasil, 2007/2008

Os pacientes foram comparados quanto à elegibilidade para o tratamento e quanto ao uso de crack na vida. A comparação dos pacientes acolhidos, no que tange à elegibilidade para o tratamento não mostrou diferenças significativas entre os grupos, como pode ser visto na Tabela 1.

Tabela 1 - Distribuição de pacientes acolhidos, conforme elegibilidade para tratamento nas variáveis estudadas. Cidade do Rio de Janeiro, RJ, Brasil, 2007/2008

\begin{tabular}{|c|c|c|c|c|c|}
\hline & \multicolumn{2}{|c|}{ Não elegíveis $n=78$} & \multicolumn{2}{|c|}{ Elegíveis n=198 } & \multirow{2}{*}{ p } \\
\hline & $f$ & $\%$ & f & $\%$ & \\
\hline \multicolumn{6}{|l|}{ Sexo } \\
\hline Masculino & 11 & 14 & 44 & 22 & 0,128 \\
\hline Feminino & 67 & 86 & 154 & 78 & \\
\hline \multicolumn{6}{|l|}{ Idade } \\
\hline $18-20$ & 7 & 9 & 17 & 9 & 0,414 \\
\hline $21-30$ & 22 & 28 & 41 & 21 & \\
\hline$>31$ & 49 & 63 & 138 & 70 & \\
\hline \multicolumn{6}{|l|}{ Escolaridade } \\
\hline Fundamental incompleto & 23 & 29 & 51 & 26 & 0,165 \\
\hline Fund. completo/médio incompleto & 18 & 23 & 49 & 25 & \\
\hline Médio completo & 17 & 22 & 45 & 23 & \\
\hline Superior incompleto/completo & 19 & 24 & 50 & 25 & \\
\hline \multicolumn{6}{|l|}{ Ocupação } \\
\hline Ativo & 27 & 35 & 70 & 35 & 0,891 \\
\hline Desempregado & 35 & 45 & 80 & 40 & \\
\hline Benefício ou aposentado & 12 & 15 & 36 & 18 & \\
\hline \multicolumn{6}{|l|}{ Estado civil } \\
\hline Solteiro & 42 & 54 & 83 & 38 & 0,771 \\
\hline Casado/vivendo como casado & 22 & 28 & 75 & 19 & \\
\hline Separado/já viveu como casado & 12 & 15 & 37 & 42 & \\
\hline \multicolumn{6}{|l|}{ Área Programática } \\
\hline Do Projad AP 2.1 & 25 & 32 & 66 & 33 & 0,942 \\
\hline Outras localidades & 52 & 67 & 132 & 67 & \\
\hline
\end{tabular}


Tabela 1 - (continuação)

\begin{tabular}{|c|c|c|c|c|c|}
\hline & \multicolumn{2}{|c|}{ Não elegíveis n=78 } & \multicolumn{2}{|c|}{ Elegíveis n=198 } & \multirow{2}{*}{ p } \\
\hline & f & $\%$ & $f$ & $\%$ & \\
\hline \multicolumn{6}{|c|}{ Tratamentos prévios } \\
\hline Sim & 31 & 40 & 94 & 47 & 0,232 \\
\hline Não & 44 & 56 & 96 & 48 & \\
\hline \multicolumn{6}{|c|}{ Grupos de mútua ajuda } \\
\hline Sim & 33 & 42 & 112 & 57 & 0,071 \\
\hline Não & 38 & 49 & 78 & 39 & \\
\hline \multicolumn{6}{|c|}{ Uso de crack na vida } \\
\hline $\operatorname{Sim}$ & 8 & 10 & 33 & 17 & 0,177 \\
\hline Não & 68 & 87 & 160 & 81 & \\
\hline \multicolumn{6}{|c|}{ Uso de crack nos últimos 30 dias $^{* *}$} \\
\hline Sim & 3 & 38 & 16 & 48 & 0,787 \\
\hline Não & 4 & 50 & 17 & 52 & \\
\hline
\end{tabular}

$* \mathrm{p} \leq 0,05 ; * *$ dentre os que fizeram uso de crack na vida

Do total de pacientes estudados, 42 (15\%) relataram uso de crack na vida e 20 (7\%) fizeram uso nos últimos 30 dias. Tendo em vista a similaridade sociodemográfica entre os elegíveis e não elegíveis para o tratamento e número relativamente pequeno de usuários de crack, distribuídos sem diferenças estatisticamente significativas entre ambos os grupos, optou-se por estudar os pacientes com relato de uso de crack na vida entre todos os acolhidos.

A comparação entre aqueles com e sem relato de uso de crack na vida mostrou que os pacientes com uso de crack são mais jovens, predominantemente solteiros. Além disso, há menor procura prévia entre os usuários de crack por grupos de mútua ajuda, como pode ser observado na Tabela 2 .

Tabela 2 - Distribuição dos pacientes acolhidos, conforme uso de crack na vida nas variáveis estudadas. Cidade do Rio de Janeiro, RJ, Brasil, 2007/2008

\begin{tabular}{|c|c|c|c|c|c|}
\hline & \multicolumn{2}{|c|}{$\begin{array}{c}\begin{array}{c}\text { Sem uso de crack } \\
n=229\end{array} \\
\end{array}$} & \multicolumn{2}{|c|}{$\begin{array}{c}\text { Com uso de crack } \\
n=42\end{array}$} & \multirow[t]{2}{*}{ p } \\
\hline & f & $\%$ & f & $\%$ & \\
\hline \multicolumn{6}{|l|}{ Sexo } \\
\hline Masculino & 183 & 80 & 34 & 81 & 0,877 \\
\hline Feminino & 46 & 20 & 8 & 19 & \\
\hline \multicolumn{6}{|l|}{ Idade } \\
\hline $18-20$ & 15 & 7 & 7 & 17 & $0,000^{*}$ \\
\hline $21-30$ & 41 & 18 & 22 & 52 & \\
\hline$>31$ & 171 & 75 & 13 & 31 & \\
\hline \multicolumn{6}{|l|}{ Escolaridade } \\
\hline Fundamental incompleto & 59 & 26 & 13 & 31 & 0,566 \\
\hline Fund.completo/médio incompleto & 56 & 24 & 11 & 26 & \\
\hline Médio completo & 51 & 22 & 11 & 26 & \\
\hline Superior incompleto/completo & 61 & 27 & 7 & 17 & \\
\hline \multicolumn{6}{|l|}{ Ocupação } \\
\hline Ativo & 82 & 36 & 13 & 31 & 0,127 \\
\hline Desempregado & 90 & 39 & 23 & 55 & \\
\hline Benefício ou aposentado & 44 & 19 & 4 & 10 & \\
\hline \multicolumn{6}{|l|}{ Estado civil } \\
\hline Solteiro & 92 & 40 & 30 & 71 & $0,001^{*}$ \\
\hline Casado/vivendo como casado & 90 & 39 & 7 & 17 & \\
\hline Separado/já viveu como casado & 44 & 19 & 5 & 12 & \\
\hline
\end{tabular}


Tabela 2 - (continuação)

\begin{tabular}{|c|c|c|c|c|c|}
\hline & \multicolumn{2}{|c|}{$\begin{array}{c}\text { Sem uso de crack } \\
n=229\end{array}$} & \multicolumn{2}{|c|}{$\begin{array}{c}\text { Com uso de crack } \\
\mathrm{n}=42\end{array}$} & \multirow[t]{2}{*}{ p } \\
\hline & $f$ & $\%$ & $f$ & $\%$ & \\
\hline \multicolumn{6}{|l|}{ Área programática } \\
\hline Do Projad (AP 2.1 & 72 & 31 & 18 & 43 & 0,160 \\
\hline Outras localidades & 155 & 68 & 24 & 57 & \\
\hline \multicolumn{6}{|l|}{ Tratamentos prévios } \\
\hline $\operatorname{Sim}$ & 106 & 46 & 19 & 45 & 0,868 \\
\hline Não & 116 & 51 & 22 & 52 & \\
\hline \multicolumn{6}{|l|}{ Grupos de mútua ajuda } \\
\hline Sim & 129 & 56 & 16 & 38 & $0,027^{*}$ \\
\hline Não & 90 & 39 & 24 & 57 & \\
\hline
\end{tabular}

$* p \leq 0,05$

Dentre os elegíveis para tratamento, o retorno ao serviço após o acolhimento foi de 74\%, entre pacientes não usuários de crack e de 79\% entre usuários $(p=0,899)$. O retorno ao serviço em ambos os grupos foi de $21 \%$.

$\mathrm{Na}$ análise, apenas, dos 42 pacientes com relato de uso de crack na vida, 20 pacientes (48\%) relatavam uso nos últimos 30 dias. Comparando-se os pacientes com e sem relato de uso nos últimos 30 dias, não foram observadas diferenças estatisticamente significativas nas variáveis estudadas. Houve tendência apenas para a ausência de procura prévia por grupos de mútua ajuda, ou outros tratamentos, entre aqueles com uso nos últimos 30 dias ( $p=0,103$ e $p=0,081$, respectivamente).

A distribuição percentual mensal de pacientes com uso de crack na vida e nos últimos 30 dias, acolhidos no serviço, é mostrada na Figura 2. Os dados mostram o crescimento da procura nos últimos meses do período avaliado.

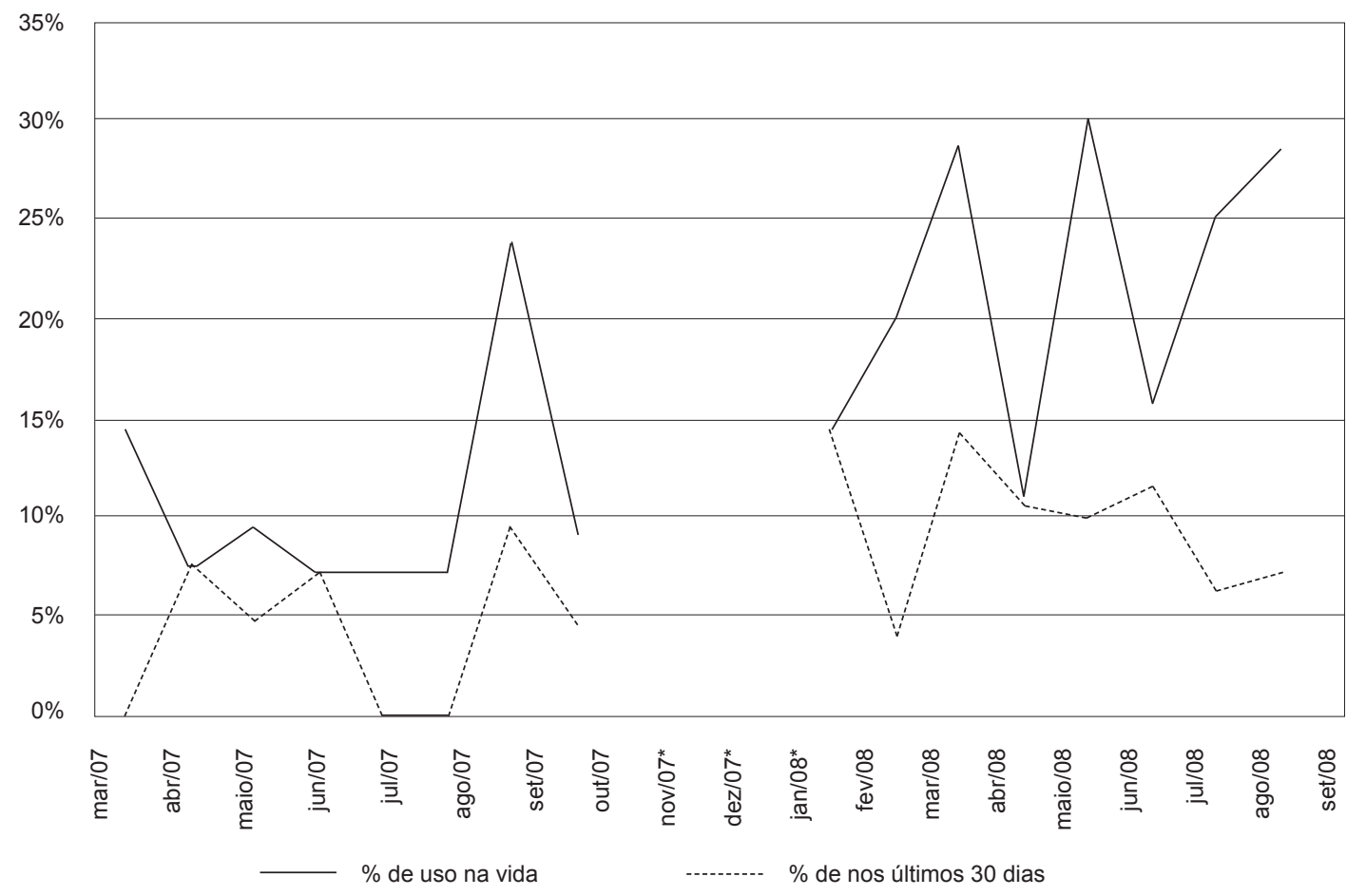

*meses sem acolhimento no Projad

Figura 2 - Distribuição percentual mensal dos pacientes que referiram uso de crack na vida e nos últimos 30 dias, em relação aos que buscam atendimento no Projad. Cidade do Rio de Janeiro, RJ, Brasil, 2007/2008 


\section{Discussão}

A similaridade das características sociodemográficas entre os pacientes elegíveis ou não para o tratamento era esperada, tendo em vista tratar-se de um centro de atenção terciário e que, mesmo seguindo as orientações gerais de regionalização do SUS, também atende casos de maior complexidade.

Estudos apontam que os usuários de crack constituem grupo distinto entre os usuários de drogas ${ }^{(9-}$ 10), de características próprias, que necessitam de abordagem especial, devido ao acelerado processo de deterioração física e psíquica a que estão $\operatorname{sujeitos}^{(7,9,13)}$.

$\mathrm{Na}$ amostra estudada, houve predomínio de homens, os usuários de crack eram significativamente mais jovens e houve maior número de solteiros em relação aos usuários de outras substâncias. O perfil desses pacientes é semelhante ao encontrado por outros estudos com usuários de $\operatorname{crack}^{(7,9-10,14-16)}$.

Entre os usuários de crack, 55\% estavam desempregados. Esse percentual é semelhante aos encontrados em estudos similares ${ }^{(7,9,15)}$. Entretanto, neste estudo, não foi encontrada diferença estatisticamente significativa entre usuários e não usuários de crack, no que se refere à ocupação.

Embora a maior proporção de pacientes com escolaridade inferior ao ensino fundamental tenha sido encontrada entre usuários de $c r a c k$, a diferença não foi significativa. A proporção de $31 \%$ dos usuários de crack que não completaram o ensino fundamental e de $43 \%$ que apresentaram escolaridade igual ou superior ao ensino médio completo reflete nível de escolaridade diferente daquele evidenciado por outros estudos, que apontam níveis significativamente mais baixos de escolaridade entre usuários de $\operatorname{crack}^{(10,14,17)}$.

Analisando-se os pacientes elegíveis para tratamento, encontrou-se número relativamente maior de usuários de crack na AP 2.1, região que abrange bairros considerados de classe média/alta. Esse dado pode indicar que os usuários de crack não são apenas moradores de rua ou de baixa renda, o que sugere que, talvez, no Rio de Janeiro, a cultura do crack seja diferente daquela observada em outras cidades do Brasil(18).

Observou-se que os usuários de crack elegíveis para tratamento no Projad, com maior frequência, não haviam procurado previamente grupos de mútua ajuda. Entre os usuários de crack, nos últimos 30 dias, também houve tendência à menor procura prévia por grupos de mútua ajuda, em relação àqueles usuários de crack sem uso, nesse período. O fato de ser população mais jovem e de consumir droga com alto poder indutor de dependência pode estar relacionado a esse achado. Outra possibilidade é de se tratar de pacientes de maior gravidade, que procuram desde o início centros de referência em atendimento especializado.

O uso de crack nos últimos 30 dias não interferiu na elegibilidade ou no retorno do paciente ao serviço. Ainda que, no presente estudo, a maioria dos pacientes tenha comparecido aos encaminhamentos efetuados, $21 \%$ dos pacientes não retornaram aos encaminhamentos feitos, evidenciando que a dificuldade de adesão se inicia entre o grupo de acolhimento e o primeiro atendimento efetivo. A literatura salienta dificuldade de usuários dessa substância em comparecer ao encaminhamento proposto(9).

Chama a atenção a tendência ao crescimento do relato de uso na vida dessa substância, ao longo do período estudado, como pode ser observado na Figura 1. No Brasil, serviços ambulatoriais especializados detectaram aumento no consumo de crack desde meados da década de 1990, porém, estudos referentes a esse crescimento no Rio de Janeiro não foram encontrados. No presente estudo, observou-se crescimento importante no número de usuários de crack, ao longo do período estudado. Embora a amostra seja pequena, esse é o primeiro estudo que documenta aumento de usuários de crack que procuraram por tratamento na cidade do Rio de Janeiro.

\section{Conclusão}

O presente estudo aponta as características de usuários de crack na cidade do Rio de Janeiro, acolhidos em serviço ambulatorial especializado. Embora predominem homens jovens e solteiros, as taxas de desemprego e baixa escolaridade não foram tão críticas quanto aquelas evidenciadas em estudos anteriores, e não diferiram dos pacientes usuários de outras substâncias.

Apesar de não ser estudo desenhado para abordar a questão do crack no Rio de Janeiro, os dados sugerem crescimento do consumo dessa substância. Este é o primeiro estudo que aponta para o crescimento de usuários de crack nessa cidade, fenômeno já descrito em outras cidades do Brasil, e que necessita ser confirmado por estudos futuros de base populacional. Esses dados podem ser utilizados como subsídios preliminares para avaliação e estruturação do serviço, fundamentando propostas de modificações na organização do atendimento ao paciente e avaliações de processos e resultados de tratamento. A extrapolação para outros serviços é limitada. 
Podem ser apontadas algumas limitações do presente estudo. Os dados sociodemográficos desta investigação foram colhidos em formulários autopreenchidos e com identificação do paciente, sem avaliação do quadro psiquiátrico no momento do preenchimento. Os pacientes foram orientados a marcar apenas uma das opções de emprego, não sendo possível, por exemplo, considerar aqueles que estão aposentados, mas realizam alguma atividade remunerada. O espaço reservado para as drogas de uso na vida e nos últimos 30 dias continha explicitado álcool, maconha e cocaína, complementado por espaço para citação de outras drogas. É possível que tenha ocorrido erros por esquecimento ou desconhecimento, incluindo o crack. Também foram observados alguns erros no preenchimento do consumo na vida e nos últimos 30 dias, utilizando-se os códigos de uso na vida para expressar uso nos últimos 30 dias e vice-versa.

\section{Agradecimentos}

Agradecemos a Comissão Interamericana para o Controle do Abuso de Drogas/CICAD da Secretaria de Segurança Multidimensional/SSM da Organização dos Estados Americanos/OEA, a Secretaria Nacional de Políticas sobre Drogas/SENAD do Gabinete de Segurança Institucional/Brasil, a Escola de Enfermagem de Ribeirão Preto da Universidade de São Paulo e o Centro Colaborador da Organização Mundial da Saúde para o Desenvolvimento da Pesquisa em Enfermagem, a população representada nas pesquisas, bem como as autoridades das universidades representadas pelos participantes do Programa On-Line de Especialização em Pesquisa sobre o Fenômeno das Drogas - períodos 2006, 2007, 2008 e 2009.

\section{Referências}

1. World Health Organization (WHO). Guide to drug abuse epidemiology, 2000. [acesso em: 03 dezembro 2009]. Disponível em: http://www.who.int/substance_ abuse/publications/epidemiology/en/index.html

2. Pan American Health Organization (PAHO) / World Health Organization (WHO). Behavioral Disorders. [acesso em: 03 dezembro 2009]. Disponível em: http:// www. paho.org/Project. asp?SEL $=$ TP\&LNG $=E N G \& I D=51$ 6\&PRGRP=docs_gen

3. Carlini EA, Galduróz JC, Noto AR, Nappo SA. I Levantamento domiciliar nacional sobre uso de drogas psicotrópicas no Brasil (2001). São Paulo: Secretaria Nacional Antidrogas/Centro Brasileiro de Informações sobre Drogas Psicotrópicas; 2002
4. Carlini EA, Galduróz JC, Noto AR, Nappo SA. II Levantamento domiciliar sobre 0 uso de drogas psicotrópicas no Brasil: estudo envolvendo as 108 maiores cidades do país: 2005. São Paulo: CEBRID - Centro Brasileiro de Informação sobre Drogas Psicotrópicas: UNIFESP - Universidade Federal de São Paulo; 2006.

5. Word CO, Bowser B. Background to crack cocaine addiction and HIV high-risk behavior: the next epidemic Am J Drug Alcohol Abuse. 1997 Feb;23(1):67-77.

6. Haydon E, Fischer B. Crack use as a public health problem in Canada: call for an evaluation of 'safer crack use kits'. Can J Public Health. 2005 MayJune; $96(3): 185-8$.

7. Ribeiro M, Dunn J, Sesso R, Dias AC, Laranjeira R Causes of death among crack cocaine users. Rev Bras Psiquiatr. 2006 Sep;28(3):196-202

8. Ferri CP, Laranjeira RR, da Silveira DA, Dunn J, Formigoni ML. Increase in the search for treatment by crack users in 2 outpatient clinics at the city of Sao Paulo from 1990 to 1993. Rev Assoc Med Bras. 1997 JanuaryMarch;43(1):25-8.

9. Duailibi LB, Ribeiro M, Laranjeira R. Profile of cocaine and crack users in Brazil. Cad Saude Publica. 2008;24 Suppl 4:s545-57.

10. Guindalini C, Vallada H, Breen G, Laranjeira R. Concurrent crack and powder cocaine users from Sao Paulo: do they represent a different group? BMC Public Health. 2006 January 20; 6:10.

11. O Globo [internet]. Quantidade de crack apreendida pela policia no estado cresceu $542 \%$ em um ano. [acesso em: 03 dezembro 2009]. Disponível em: http://oglobo. globo.com/rio/mat/2009/10/27/quantidade-de-crackapreendida-pela-policia-no-estado-cresceu-542-emum-ano-914396790.asp

12. Cruz MS. O crack e sua espiral compulsiva. O Estado de São Paulo (Grupo Estado Ed.). 2009, 12 julho, Seção Aliás, p. 15.

13. Cunha PJ, Nicastri S, Gomes LP, Moino RM, Peluso MA. Neuropsychological impairments in crack cocainedependent inpatients: preliminary findings. Rev Bras Psiquiatr. 2004 June;26(2):103-6.

14. Ferreira OF Filho, Turchi MD, Laranjeira R, Castelo A. Epidemiological profile of cocaine users on treatment in psychiatrics hospitals, Brazil. Rev Saude Publica. 2003 December;37(6):751-9.

15. Ferri CP, Gossop M. Route of cocaine administration: patterns of use and problems among a Brazilian sample. Addict Behav. 1999 Nov-Dec;24(6):815-21.

16. Passos SR, Camacho LA. Client characteristics at a center for treatment of drug dependence. Rev Saude Publica. 1998 February;32(1):64-71. 
17. Miech RA, Chilcoat $H$, Harder VS. The increase in the association of education and cocaine use over the $1980 \mathrm{~s}$ and 1990s: evidence for a 'historical period' effect. Drug Alcohol Depend. 2005 September 1;79(3):311-20.

18. Oliveira LG, Nappo SA. Characterization of the crack cocaine culture in the city of São Paulo: a controlled pattern of use. Rev Saude Publica. 2008 August;42(4):664-71. 